\title{
Total arterial coronary artery bypass grafting of multiple coronary aneurysms
}

\author{
Thin Xuan Vo, MD, MSc, Janet Mee Chin Ngu, MD, MSc, and David Glineur, MD, PhD, Ottawa, Ontario, \\ Canada
}

From the Division of Cardiac Surgery, University of Ottawa Heart Institute, Ottawa, Ontario, Canada. Disclosures: The authors reported no conflicts of interest.

The Journal policy requires editors and reviewers to disclose conflicts of interest and to decline handling or reviewing manuscripts for which they may have a conflict of interest. The editors and reviewers of this article have no conflicts of interest.

Received for publication April 16, 2021; accepted for publication April 20, 2021; available ahead of print April $28,2021$.

Address for reprints: David Glineur, MD, PhD, University of Ottawa Heart Institute, 40 Ruskin Street, Ottawa, Ontario, Canada K1Y 4W7 (E-mail: DGlineur@ottawaheart.ca).

JTCVS Techniques 2021;9:73-7

2666-2507

Copyright (C) 2021 The Authors. Published by Elsevier Inc. on behalf of The American Association for Thoracic Surgery. This is an open access article under the CC BY-NC-ND license (http://creativecommons.org/licenses/bync-nd/4.0/).

https://doi.org/10.1016/j.xjtc.2021.04.016

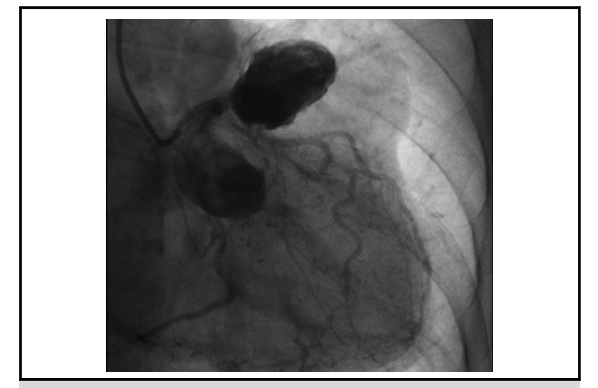

Coronary angiogram revealing giant coronary artery aneurysms of the left coronary system.

Giant coronary artery aneurysms (GCAAs) are rare, with a reported prevalence of $0.02 \%$ of aneurysms with a diameter $>50 \mathrm{~mm}{ }^{1}$ A threshold of $50 \mathrm{~mm}$ has been used to define GCAA in some studies, ${ }^{1,2}$ while others suggest a diameter of $\geq 4$ times the reference vessel size or $\geq 20 \mathrm{~mm}$. Most coronary artery aneurysms are asymptomatic and are found incidentally. ${ }^{3}$ Complications of untreated coronary aneurysms include thromboembolism, spontaneous rupture, myocardial ischemia/infarction, compression, and fistula formation. ${ }^{3}$ We report the surgical management of a patient who was found to have several large coronary aneurysms in all major coronary territories.

\section{CASE DESCRIPTION}

A 74-year-old woman without previously known cardiac disease presented with syncope and stable angina. She did not have a history of connective tissue disease or vasculitis. She had a history of infrarenal abdominal aortic aneurysm $(3.4 \mathrm{~cm})$ and a mildly dilated ascending aorta $(3.9 \mathrm{~cm})$. Coronary angiography revealed several large coronary aneurysms in all coronary territories with stagnant flow noted in GCAAs of the left anterior descending artery (LAD) and left circumflex artery (LCx) (Figure 1, Video 1, Video 2, Video 3). Computed tomography angiography (CT-A) revealed a GCAA on her LAD $(5.5 \times 4.8 \mathrm{~cm})$ and $\mathrm{LCx}$ $(3.9 \times 2.7 \mathrm{~cm})$. She had a smaller coronary aneurysm at the distal right coronary artery $(\mathrm{RCA})(1.8 \times 1.2 \mathrm{~cm})$ and

a small coronary aneurysm at the first obtuse marginal $(\mathrm{OM})$ measuring $0.7 \times 0.9 \mathrm{~cm}$ (Figure 2, Video 4). CT-A also revealed stenosis of the LAD (70\%) and RCA $(70 \%)$ proximal to their respective aneurysms. Transthoracic echocardiography revealed normal valves and normal biventricular function.

A multidisciplinary discussion was held, and several options were considered, including aneurysm coiling, covered stent placement, and coronary artery bypass grafting (CABG). Given the diffuse nature of the aneurysms, complex anatomy, and risk of rupture, a unanimous decision was made to pursue surgery, and the patient was consented. The Institutional Review Board at the University of Ottawa Heart Institute did not approve this study, as this approval is not required for case reports. The patient provided informed written consent for publication and dissemination of her anonymized clinical and radiographic information.

Intraoperatively, owing to the size, location, and diffuse calcification of the LAD GCAA, an epicardial approach to open or occlude the LAD proximal to the aneurysm was 


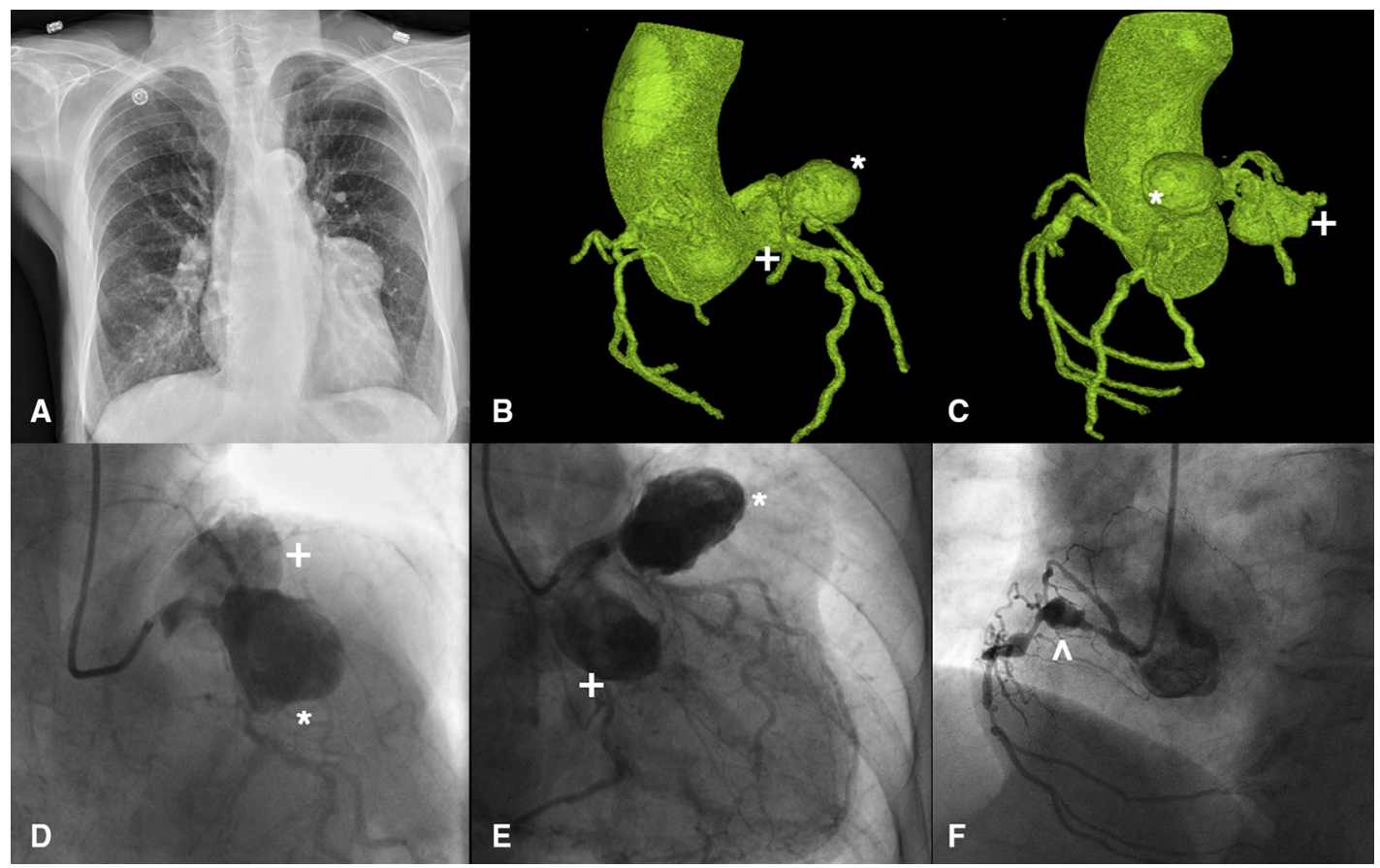

FIGURE 1. Preoperative imaging of giant coronary artery aneurysms in a 74-year-old woman presenting with angina and syncope. A, Chest X-ray showing the calcified outline of left anterior descending giant coronary artery aneurysm. B and C, 3-dimensional computed tomography scan reconstruction emphasizing left anterior descending (LAD) aneurysm and left circumflex (LCx) aneurysms in relation to each other. D, Coronary angiogram with anteroposterior view of leftsided giant coronary aneurysms. E, Coronary angiogram with right anterior oblique view of left-sided giant coronary aneurysms. F, Coronary angiogram with view of the right coronary artery (RCA) with multiple aneurysms and distal RCA stenosis. *LAD aneurysm; +, LCx aneurysm;, RCA aneurysm.

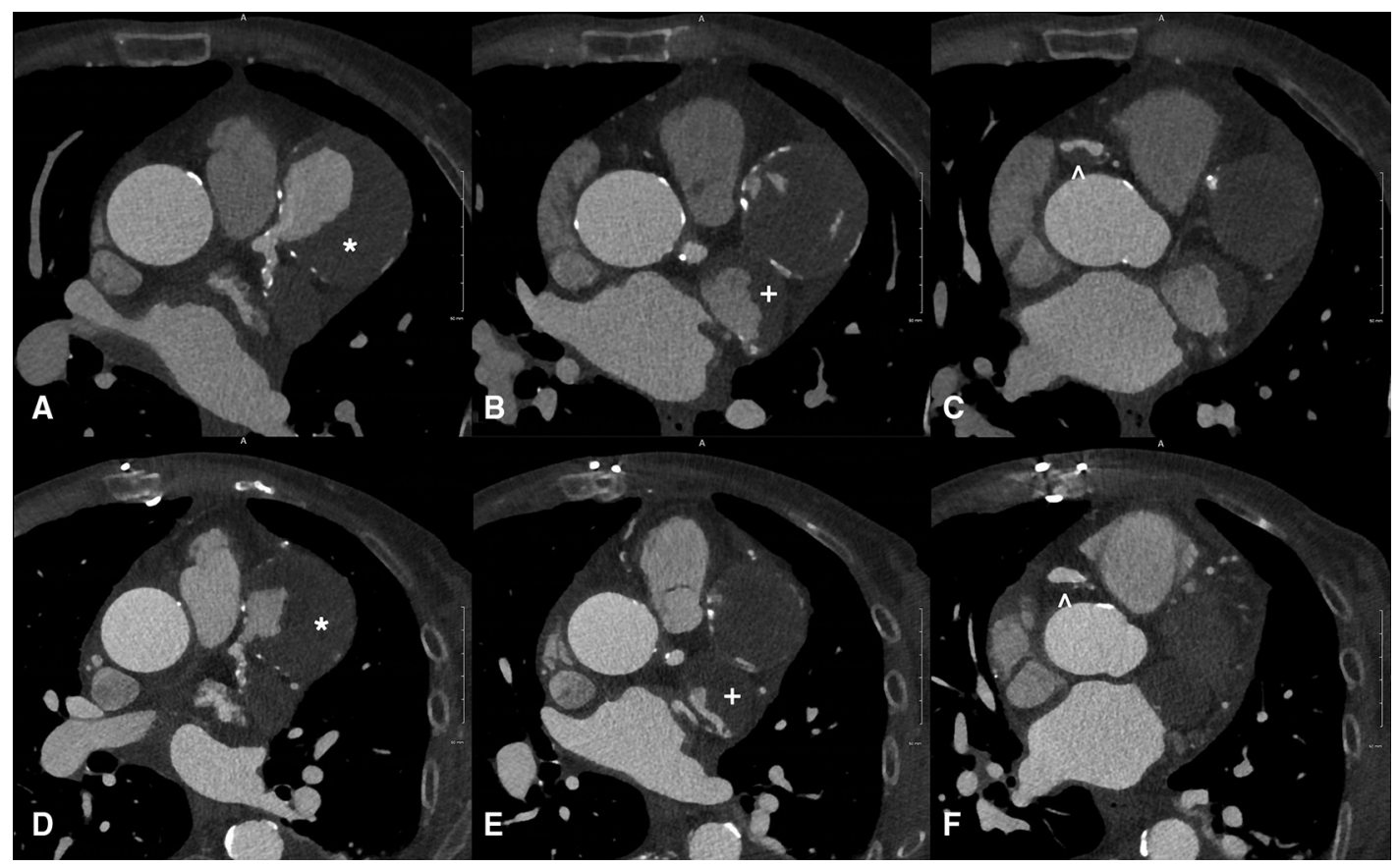

FIGURE 2. Computed tomography angiogram of preoperative (A, B, C) and 3-month postoperative (D, E, F) giant coronary aneurysms in a 74-year-old woman presenting with angina and syncope. A. Left anterior descending (LAD) giant coronary aneurysm with largest dimension of $5.5 \times 4.8 \mathrm{~cm}$. B, Left circumflex (LCx) giant coronary aneurysm with largest dimension of $3.9 \times 2.7 \mathrm{~cm}$. C, Distal right coronary artery (RCA) aneurysm with largest dimension of $1.8 \times 1.2 \mathrm{~cm}$. D, LAD thrombosed giant coronary aneurysm with largest dimension of $5.4 \times 4.9 \mathrm{~cm}$. E, LCx thrombosed giant coronary aneurysm with largest dimension of $3.8 \times 2.7 \mathrm{~cm}$. F, Distal RCA aneurysm with maximal dimension of $1.7 \times 1.1 \mathrm{~cm}$. *LAD aneurysm;,$+ \mathrm{LCx}$ aneurysm; $\hat{,}$ RCA aneurysm. 


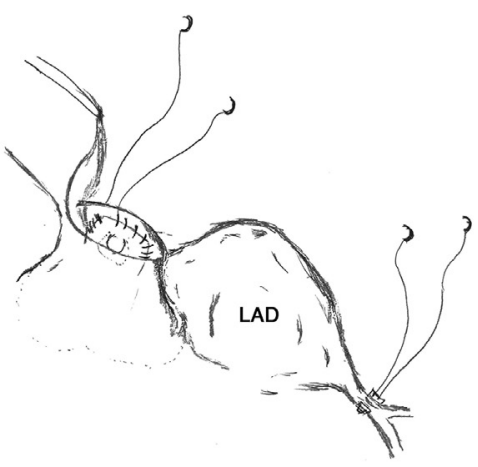

A

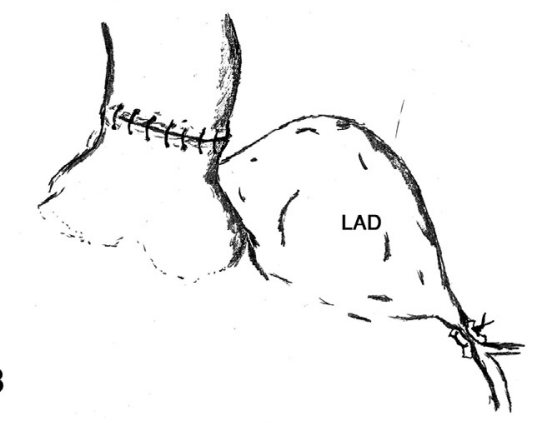

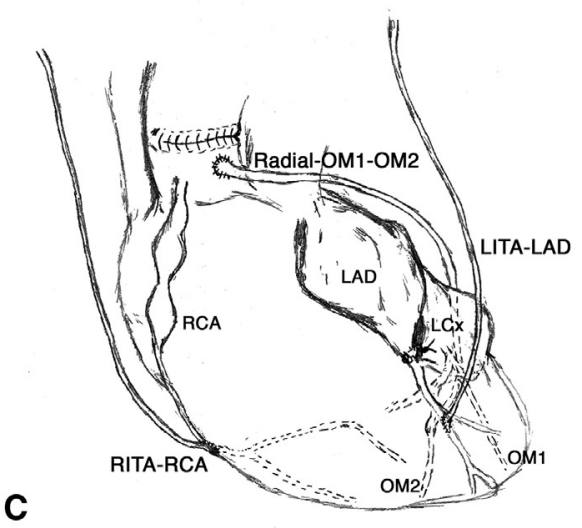

C

FIGURE 3. Schematic of operative management of giant coronary aneurysms in a 74-year-old woman presenting with angina and syncope. A, Occlusive patch fashioned from bovine pericardium to patch over the left main coronary ostia using a running Prolene suture via an aortotomy. This was done to decrease proximal pressurization of the left coronary system aneurysms. A pledgeted Prolene suture was also used to occlude the left anterior descending artery $(L A D)$ aneurysm distally. B, Completion of aortotomy closure and LAD ligated distal to the aneurysm. C, Coronary artery bypass grafting configuration to supply all major coronary territories affected by coronary aneurysms and atherosclerosis. The in situ right internal thoracic artery (RITA) was anastomosed to the distal right coronary artery $(R C A)$. The in situ left internal thoracic artery (LITA) was anastomosed to the mid-distal LAD. The radial artery was anastomosed from the aorta to the first obtuse marginal artery $(O M 1)$ and second obtuse marginal artery (OM2) in sequential fashion.

deemed too risky. Alternatively, to occlude proximal flow, a small piece of pericardium was fashioned and sutured over the left main ostia from the inside via aortotomy (Figure 3). The LAD GCAA was occluded distally with a pledgeted Prolene suture. The LCx GCAA was difficult to address because of its location in the left atrial groove and was not ligated, to avoid inadvertent injury. All of the territories were bypassed with LITA-LAD, radial artery from the aorta to OM1 with sequential to OM2, and in situ RITA-RCA. The patient had an uneventful postoperative course.

She was seen 2 months postoperatively, demonstrating resolution of her angina and no further episodes of syncope. Follow-up CT-A at 3 months revealed patent grafts with subtotal thrombosis of the aneurysms (Figure 2, Video 5). A CT scan of the head did not reveal any evidence of cerebral aneurysms. She remained angina and syncope-free at 3year follow-up.

\section{DISCUSSION}

We report on a rare case of multiple coronary artery aneurysms of all major coronary territories, including GCAAs in the LAD and LCx, with successful surgical repair. Most GCAAs are seen in the RCA rather than the left-sided coronaries. ${ }^{2,4}$ Aneurysms in more than one area occur in only approximately $25 \%$ of cases when a coronary aneurysm $>5 \mathrm{~cm}$ is present. ${ }^{4}$

GCAAs can be managed medically with antiplatelets and/or anticoagulation, percutaneous intervention, or surgical repair. ${ }^{3}$ Different approaches to surgical repair have been reported, including CABG, arteriotomy with thrombectomy, ligation/plication, and patch repair. ${ }^{1,2,5}$ Here we used a multifaceted approach with an occlusive patch, ligation, and CABG. Our approach avoided cutting into the aneurysm, decreasing the risk of emboli. However, without opening the aneurysm to occlude smaller feeding vessels, there remains the risk of residual luminal filling and "endotension." Nonetheless, the risk of rupture is decreased due to decreased pressurization and ongoing thrombosis, fibrosis, and calcification. Complete arterial revascularization was performed for the possibility of improved long-term patency given the patient's excellent preoperative functional status and anticipated lifespan. 


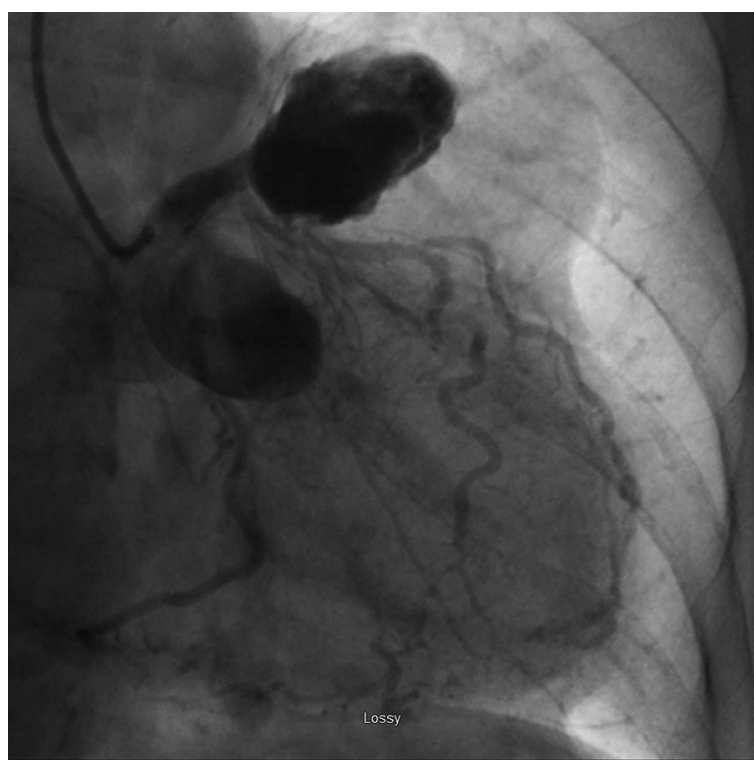

VIDEO 1. Coronary angiogram cine of the left coronary system (right anterior oblique caudal view) depicting giant coronary aneurysms of the left anterior descending (LAD) artery and left circumflex artery (LCx). There is stagnant flow and retained angiographic dye in the giant coronary aneurysms. Note the radiographic shadows that indicate the true size of the aneurysms. The discrepancy between the radiographic shadow of the aneurysms and visualized flow within the aneurysms reflects existing thrombosis within the aneurysms. Video available at: https://www.jtcvs.org/ article/S2666-2507(21)00311-4/fulltext.

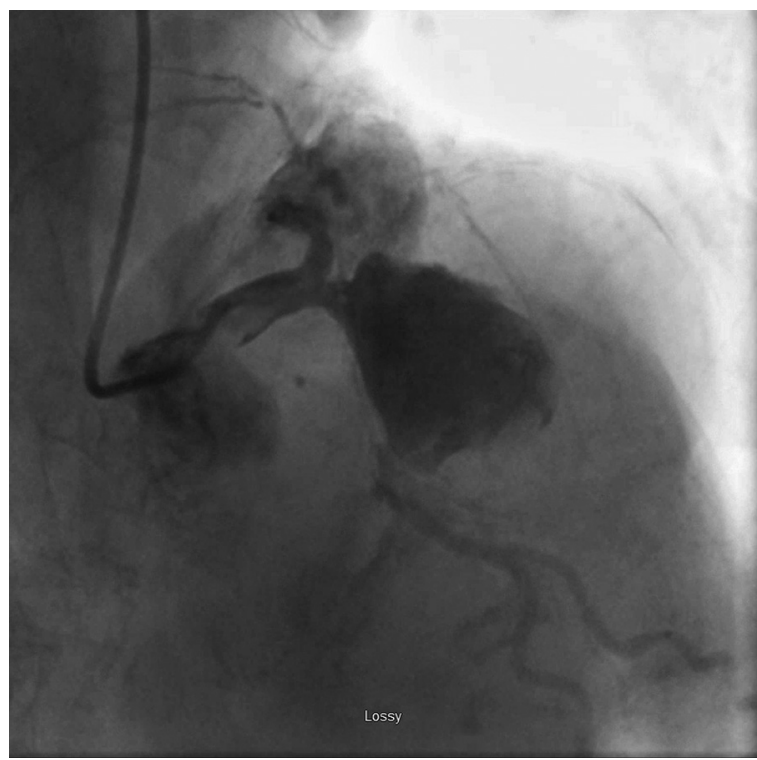

VIDEO 2. Coronary angiogram cine of the left coronary system (right anterior oblique cranial view) depicting two giant coronary aneurysms of the left anterior descending (LAD) artery and left circumflex artery $(\mathrm{LCx})$. There is stagnant flow and retained angiographic dye in the giant coronary aneurysms. Note the radiographic shadows that indicate the true size of the aneurysms. The discrepancy between the radiographic shadow of the aneurysms and visualized flow within the aneurysms reflects existing thrombosis within the aneurysms. Video available at: https://www. jtcvs.org/article/S2666-2507(21)00311-4/fulltext.

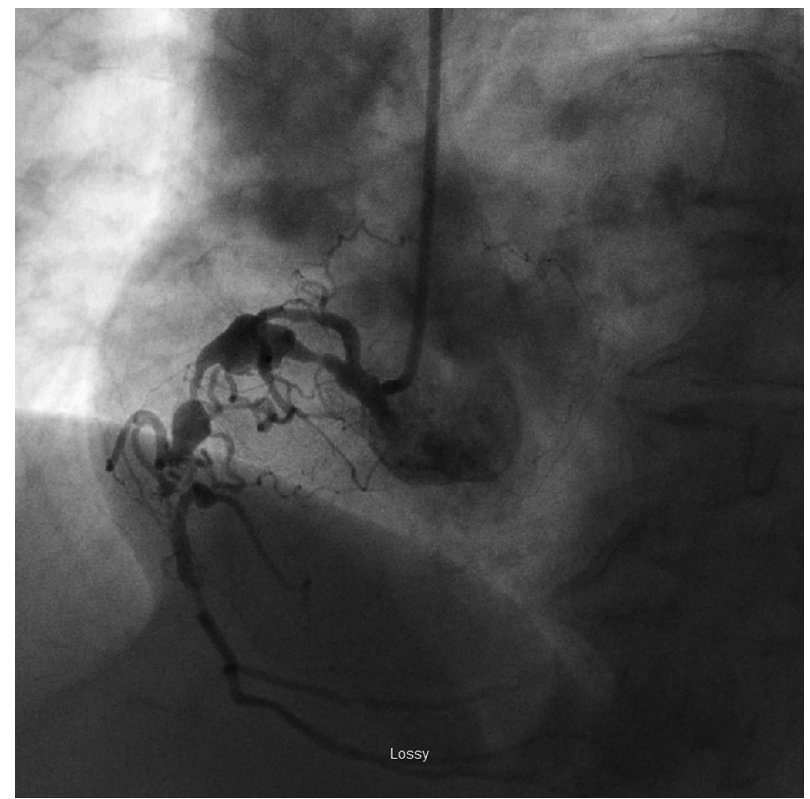

VIDEO 3. Coronary angiogram cine of the right coronary system (left anterior oblique view) depicting 2 coronary aneurysms in the right coronary artery (RCA). In addition to the 2 large sequential aneurysms in the RCA, there is a moderately stenotic lesion prior to the first RCA aneurysm and a highly stenotic lesion distal to the second RCA aneurysm. Video available at: https://www.jtcvs.org/article/S2666-2507(21)00311-4/fulltext.

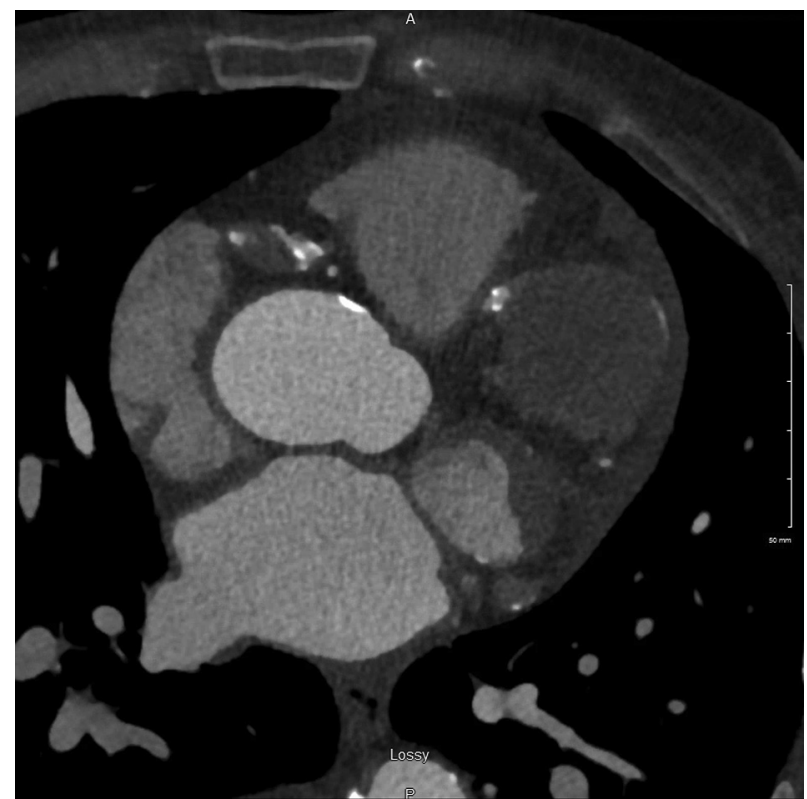

VIDEO 4. Preoperative computed tomography angiogram cine (axial plane) of multiple coronary aneurysms in a 74-year-old woman presenting with angina and syncope. There are giant coronary artery aneurysms on the left anterior descending (LAD) coronary artery and left circumflex (LCx) coronary artery. There are 2 large coronary artery aneurysms on the right coronary artery (RCA). There is also a coronary artery aneurysm seen on the first obtuse marginal artery. Video available at: https://www.jtcvs.org/ article/S2666-2507(21)00311-4/fulltext. 


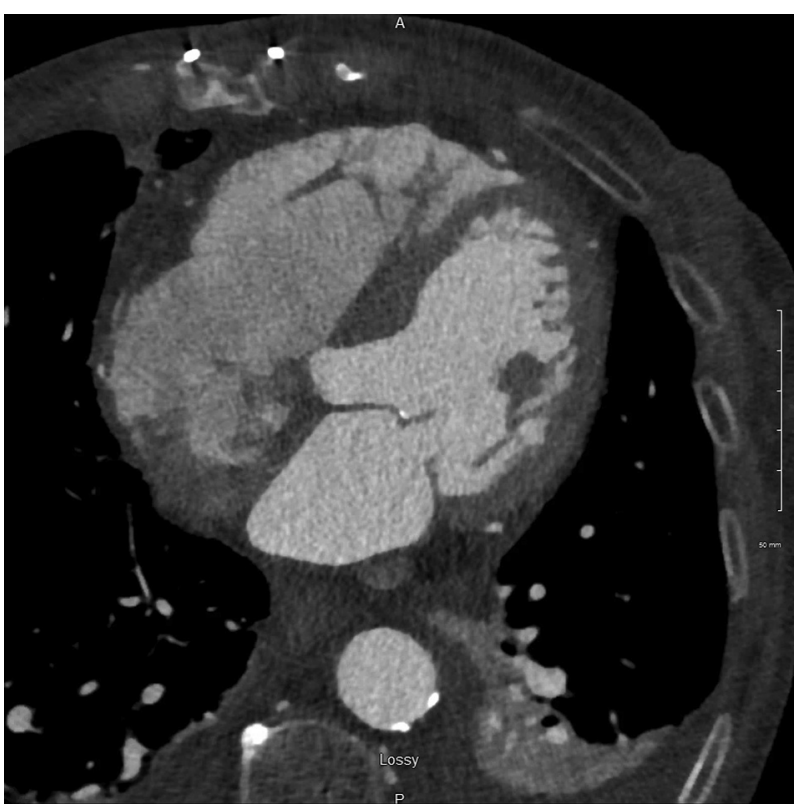

VIDEO 5. Postoperative (3-month) computed tomography angiogram cine (axial plane) of multiple coronary aneurysms in a 74-year-old woman presenting with angina and syncope. There are giant coronary artery aneurysms on the left anterior descending (LAD) coronary artery and left circumflex (LCx) coronary artery. The giant coronary artery aneurysms are largely thrombosed with minimal luminal filling within the aneurysms. There are 2 large coronary artery aneurysms on the right coronary artery (RCA). There is also a coronary artery aneurysm seen on the first obtuse marginal (OM) artery. The coronary artery bypass grafts remain patent. The in situ right internal thoracic artery (RITA) is anastomosed to the distal RCA, and the in situ left internal thoracic artery (LITA) is anastomosed to the LAD. The left radial artery travels from the aorta proximally to the first and second $\mathrm{OM}$ arteries in sequential fashion. The sizes of the coronary artery aneurysms are comparable to the preoperative images. Video available at: https://www.jtcvs.org/article/S2666-2507(21)00311-4/fulltext.
Although previous studies have investigated multiarterial grafting in coronary artery aneurysms, this is the first known report in the literature demonstrating complete arterial revascularization in the context of multiple GCAAs. ${ }^{5}$ Currently, evidence is lacking for any superior surgical approach to coronary aneurysms. Data regarding graft patency in this setting are also limited. Certain features of coronary artery aneurysms warrant surgical consideration: left main aneurysms, multiple coronary aneurysms, GCAAs, fistulas, structural compression, and saphenous vein graft aneurysms. ${ }^{3}$ Surgical intervention with focus on attenuating flow to the aneurysm(s) and revascularization of at-risk myocardium remains a safe and viable option for the definitive management of coronary aneurysms.

\section{References}

1. Li D, Wu Q, Sun L, Song Y, Wang W, Pan S, et al. Surgical treatment of giant coronary artery aneurysm. J Thorac Cardiovasc Surg. 2005;130:817-21.

2. Crawley P, Mahlow WJ, Huntsinger DR, Afiniwala S, Wortham DC. Giant coronary artery aneurysms. Tex Heart Inst J. 2014;41:603-8.

3. Kawsara A, Núñez Gil IJ, Alqahtani F, Moreland J, Rihal CS, Alkhouli M. Management of coronary artery aneurysms. JACC Cardiovasc Interv. 2018;11: 1211-23.

4. Keyser A, Hilker MK, Husser O, Diez C, Schmid C. Giant coronary aneurysms exceeding $5 \mathrm{~cm}$ in size. Interact Cardiovasc Thorac Surg. 2012;15:33-6.

5. Beckmann E, Rustum S, Marquardt S, Merz C, Shrestha M, Martens A, et al. Surgical treatment of coronary artery aneurysms. J Card Surg. 2017;32:674-9. 